\title{
BPM Tools for Asset Management in Renewable Energy Power Plants
}

\author{
Carchiolo Vincenza*, Catalano Giovanni ${ }^{\ddagger}$ Malgeri Michele ${ }^{\dagger}$, Pellegrino Carlo ${ }^{\dagger}$, Platania Giulio $^{\dagger}$, Trapani Natalia ${ }^{\dagger}$, \\ * Dipartimento di Matematica ed Informatica - Universitá di Catania - Catania - Italy \\ $\dagger$ Dipartimento di Ingegneria Elettrica Elettronica Informatica - Universitá di Catania - Catania - Italy \\ $\ddagger$ Development and Support Center - BaxEnergy - Catania, Italy
}

\begin{abstract}
Business Process Management (BPM) is an accepted discipline and its importance in increasing automation inside industrial environment is today recognized by all players. The complexity of modern management process will lead to chaos without a well-designed and effective BPM. Several BPM Suites were compared and BPM approach was applied to the case study of process management in a renewable energy power plant. Results both in process reduction and simplification and flow optimization obtained in the real case are discussed to state efficacy and efficiency of the adopted approach.
\end{abstract}

\section{INTRODUCTION}

$\mathbf{I}$ $\mathrm{N}$ A competitive environment in which companies have to provide more and more effective and efficient services/products, asset management allows to obtain value from assets and achieve the company's business objective [1]. To accomplish both the goals to ensure good operational performance and long durability of the final products/services, it is necessary to define effective business processes, to monitor their performances and to provide corrective actions when necessary. In fact, the quality of the operational processes are more and more dependent on maintenance processes, thus they must be carefully engineered and effectively implemented. Maintenance management changed in the last decades thanks to ICT development, from a management perspective, Computerized Maintenance Management System (CMMS) has contributed to enhance the control of maintenance activities [2] and maintenance is considered a relevant business function, able to interact with all other strategic functions, such as operations, purchasing, suppliers (service providers), warehouses management, administration, therefore maintenance effectiveness and efficiency is crucial for business.

BPM frameworks provide tools that allow to design the business process using model, maps and rules, than it allows to implement them defining the architecture and adding rules. Moreover, framework executes and monitors the process (providing processes measurement) collects data, and, finally, it provides analysis and diagnosis that allows to improve the process itself [3].

Indeed, the process can be viewed from different perspectives thus producing multiple models and styles [4]: control flow perspective, resource perspective that means to focus on equipment, units, etc.; data creation perspective; time perspective that focuses on deadlines and function perspective that uses activities.
BPMN 2.0 is a logical description of business processes and how they operate that focuses on process implementation and Process simulation [5]. Usually, the business process simulation collects several data and provide a visual animation of the evolution of the process. The analysis of data permits the designer together with supervisor and manager to identify (potentially expensive) mistakes and, thanks to the Key Performance Indicators (KPIs), defined during the early designing of the process, increase the performance to getting and overall improvement of the process (e.g. removing bottlenecks)

In literature, the use of the BPM approach for maintenance management is not so common [6] (see references in [7], [8]). Most of them suggest that an Asset Management System Software can help organizations to achieve operational excellence, through a more effective cost control, a more efficient asset planning, a reducing in capital expenses, an optimization of operational costs, thus extending asset life-cycle and obtaining a higher Return On Asset.

This works briefly presents some of the criteria that were adopted by BaxEnergy (c) to develop a BPMS, starting from existing platform aiming at optimizing the maintenance of renewable energy power plants to be offered as a service to different companies. Renewable-energy Power Plant faces different challenges [9], [10], [11]. Finally, a case study is discussed highlighting the enhancement and discussing some details that lead to final implementation.

Section II introduces BPMS and some of the criteria used to compare the platforms, and provide the reader with a comparison among several platforms that was available at the moment of the study. Section III discussed the case study developed using the selected platform.

\section{BPMS COMMON FEATURES AND CRITERIA}

BPMN can be useful both for planning complex business processes and to control and monitoring them once deployed. A business process can be defined as a set of connected activities that create value for customers; usually, they are classified into three groups: strategic, operational and support. A BPMN provides users and developers with tools that clearly denote the objective, who is in charge of any operation, starting and ending points, input and outputs, constraints and monitoring points. One of the most important aspect of BPMN is surely the graphical model that, usually, is formally 
documented thanks to UML (Unified Modelling Language) class diagrams [5].

BPMN 2.0 is the current standard that adds the following characteristics: human-readability, that is a standard visual notation for modelling processes; accessibility, that means that all actors must understand what is represented; machinereadability, that implies the use of the XML notation for simulating and executing processes [12].

BPMN Graphical representations use several standard symbols that must be easily understood by both developers and users the most important are: swim lane, pool, box, event, task.

A fundamental element to make dynamic the process diagram is gateway: it allow the designer to choose where the flow of a diagram must follow a path or another by evaluating a condition. Two types are defined in the standard: XOR or Exclusive. Gateway is the task that drives the evolution of a process by evaluating a condition and selecting the next task, $O R$ or Parallel Gateway split the evolution into two parallel branches that must be joined with the same element when the evolution of the process does not support parallel activities.

\section{A. Evaluation Criteria}

There is no doubt that some interesting features for a Software developer are meaningless by the point of view of a Business Process developer, and are meaningless for a standard employee, therefore the evaluation Criteria are grouped into two classes. The first group deals with the feature useful to software developers, the most important are as follows:

- OpenSource, software suite refers to open source model, that means that products are released under an opensource license, this feature allows to inspect the source and to add any additional features;

- Community, the community supporting the software suite should be as large as possible to share problems and their solutions. A large community implies Documentation and Tutorial are easy to find and this is useful both for software and model developers;

- BPMN 2.0 supported, this is a must for any new software, since today BPMN 2.0 standard is largely supported;

- Additional modules and connectors, they permits to extend the functionality of the Software and to write new custom connectors;

- API provided, this can be useful to easily add functionalities to the BPMS.

- Innovation, of course, it is important that software solution supports all recent technologies and methodologies but it must be also "mature" (according, for instance, with Gartner Magic Quadrant [7]). Some examples of innovative features are mobile device deployment without the need to develop a dedicated application, low-code Platforms, integration with Artificial Intelligence for performance analytics and so on;

Second group of features, that we could call Functionalitybased evaluation criteria, belongs to Business Process Developers that focus on the processes themselves and, usually, have no specific experience in software development. They need to model, describe and test production processes aiming at optimizing them according to some metrics (e.g. cost, duration, response time) often conflicting. The most important are listed below:

- Web Modeler/Collaboration enabled, it enables collaboration between developers;

- Template Library, the presence of a library of reusable models is a valuable add-on to any Suite;

- Model and Process Versioning, since processes run for long times, the ability to control the current version of Models and the ability to roll back to a previous version are very useful;

- Powerful graphic interface, the interface should support advanced interface functions as, for instance, Drag\&Drop of models/processes, Form Editing that allows final users to create and edit data, to customize the colors of element in a diagram to make it more readable;

- Process deployment, the system should allows to make an instance of the modelled process to check errors and to locate performance indicators or failures;

- Testing and Simulation, the presence of an engine for simulating the process and/or validate the model is an essential feature;

- Customizable Properties, that means the ability to change the properties of the trial modelling tool.

- Business rule engine and activity Monitoring, possibility to integrate business rules for the process and to monitor the execution of a process;

- Integration with Cloud: since some BPMS are integrated with cloud service, it is important to support the integration between BPMS and Cloud.

- Role Based Security, that allows to manage of the security rules for each role.

\section{B. A Brief Survey of BPMNs Platforms}

The evaluation criteria used by throughout this work are based on Critical Capabilities such as Interaction Management, Monitoring and Business Alignment, Rules and Decision Management, Analytic and they are evaluated on the base of some Use Cases. Great importance is given to Continuous Process Improvement (referred as CPI) and to Citizen Developer Application Composition (that is the ability to leave aside from IT development staff) [5].

The BPM suites, that was evaluated, support highly intelligent applications which integrate more-advanced decision automation technologies (e.g. predictive analytic, artificial intelligence) and decision support for knowledge workers to automate business processes that require an adaptive behaviour [5]. It means that actually $i B P M s$ is not a simple modelling software or a validation engine for BPMN, but it is both bound and integrated with other functionalities that keeps track of statistics and, sometimes, let even the user to define performance indicators.

The main characteristics of the tools were summarized and compared in tables I and II, the former deals with features 
useful to developers and the latter to features useful to users, i.e. the provided functionalities.

TABLE I

CRiteria RElated to DEVElopment

\begin{tabular}{l|c|c|c|c} 
& OpenSorce & Community & Innovation & API \\
\hline BPMN.IO & Y & - & Y & - \\
Cawemo & - & - & Y & - \\
Camunda & Y & Y & Y & Y \\
Bizagi & - & Y & Y & Y \\
Appian & - & Y & Y & Y \\
Activiti & Y & Y & Y & Y \\
IBM & - & Y & Y & Y \\
jBPM & Y & Y & Y & Y \\
KissFlow & - & Y & Y & Y \\
QuickFlow & - & - & - & -
\end{tabular}

TABLE II

CRITERIA RELATED TO FUNCTIONALITY

\begin{tabular}{l|c|c|c|c} 
& Web Functions & Versioning & Testing & Monitoring \\
\hline BPMN.IO & P (Partial) & - & - & - \\
Cawemo & $\mathrm{P}$ & - & - & - \\
Camunda & $\mathrm{Y}$ & $\mathrm{Y}$ & $\mathrm{P}$ & $\mathrm{Y}$ \\
Bizagi & $\mathrm{Y}$ & $\mathrm{Y}$ & $\mathrm{Y}$ & $\mathrm{Y}$ \\
Appian & $\mathrm{Y}$ & $\mathrm{Y}$ & $\mathrm{Y}$ & $\mathrm{Y}$ \\
Activiti & $\mathrm{Y}$ & $\mathrm{Y}$ & - & $\mathrm{Y}$ \\
IBM & $\mathrm{Y}$ & $\mathrm{Y}$ & $\mathrm{Y}$ & $\mathrm{Y}$ \\
jBPM & $\mathrm{Y}$ & $\mathrm{Y}$ & $\mathrm{Y}$ & $\mathrm{Y}$ \\
KissFlow & $\mathrm{Y}$ & - & - & - \\
QuickFlow & $\mathrm{Y}$ & - & - & (SalesForce)
\end{tabular}

Since the use of opensource platform covering all the process, from development to monitoring, exposing a flexible ReST API to integrate easier with existing software are the most important requisites the selected platform was Camunda. The lack of processes Simulation is overcome thanks to the a great support of Javascript libraries that can be integrated with no effort.

\section{CASE STUdY}

\section{A. The Company}

The case study was developed, during the research project WEAMS, in an Austrian company that is a BaxEnergy (c) client. The Company's core business is the construction and operations management of wind turbine power plants. The Company produces electrical energy through wind turbines, by itself or cooperating with other partners or investors. In the case study was considered that it actually deals with operations and maintenance processes of wind turbine farms.

After studying and analyzing their general organizational structure, we find 92 different relevant processes. These processes (e.g., tool provisioning, maintenance execution in harsh weather conditions, invoicing, checking financial guarantees, executing training, and so on) include several tasks with their relevant costs. In general we identified two main process categories related to wind turbines maintenance: Core processes (usually technical ones) and Support processes (usually organizational and financial ones).

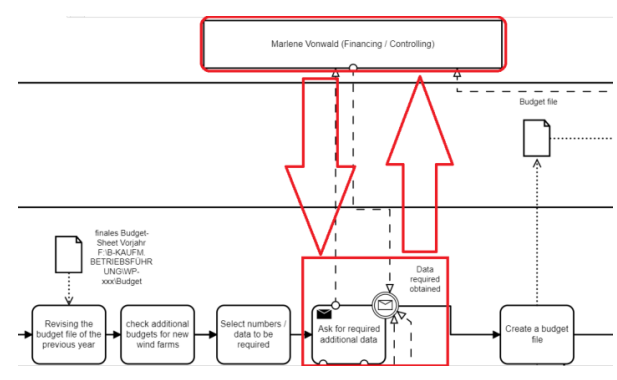

Fig. 1. Collaboration between actors of a process

\section{B. Process Re-engineering}

A subset of 39 processes out of the 92 relevant for the company were selected as they are the most frequently activated during a year and relevant also for other companies in the same business sector. Moreover, other ten maintenance core-processes, not previously engineered by the company were identified and implemented in order to guarantee better maintenance performance.

A first revision of the processes allowed to increase the general comprehension of process workflows, by the Company. In particular, to follow the correct logic of BPMN 2.0:

- The use of send and receive task was corrected;

- Some End event were added to complete some workflows;

- Some Intermediate events instead of useless tasks were introduced in order to simplify some process;

- Some script tasks were modified into normal tasks (because scripts will be added in a future phase);

- The use of gateway was corrected and useless ones were eliminated.

Furthermore, some specific processes were deleted as standalone processes and they were incorporated within other ones, by producing a unique layout representing the whole activity without missing any essential information and better visualizing interactions through inputs and outputs.

This process synthesis generated a total incorporation of 7 process into other ones, thus further reducing to 26 processes out of 39 .

Let us note, the presence of a particular interaction between pools and collapsed pools inside the processes. Those interactions called swim lanes in the BPMN 2.0 standard are used mostly to enable the collaboration between core and supporting parts of the processes.

For example, in the process Budgeting a supervisor (financing/controlling) of the process needs to receive some additional information before going on with next task. In this case, the supervisor has to do two actions (see Figure 1): Before the re-engineering, the company used the above descripted interaction also to communications with the ticket system of WEAMS. Thanks to the BPM system, implemented in the WEAMS system, this use of send/receive tasks is no more necessary (see Figure 2) but it requires just a simple task in WEAMS system (see Figure 3). In this way, there is a 


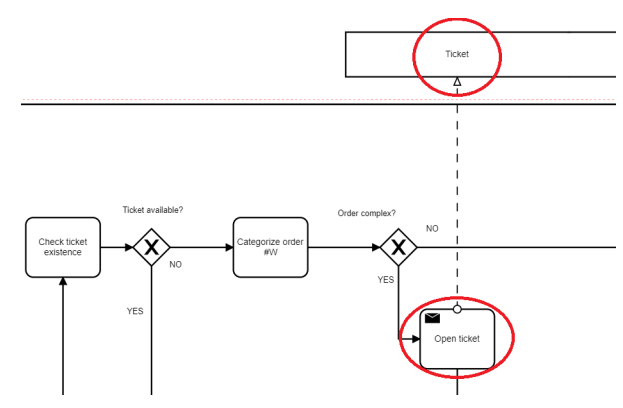

Fig. 2. Collaboration with the ticketing system of WEAMS

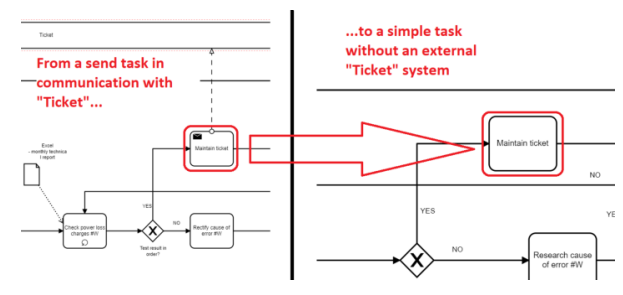

Fig. 3. From ticket system to a simple task

reduction of about 70 message flows due to ticket system implemented on the BPM system in WEAMS.

These relevant 26 processes were subjected to a deep revision in order to optimize elements and workflows and simplfy them. Such a revision allowed to eliminate from the processes 26 phases and 106 transitions that was useless, obtaining more efficacy and efficiency in process workflow and improving the general performances of the maintenance processes.

In the second step of revision some of the 26 processes were splitted into two or more sub-processes in order to easily implement them into the WEAMS BPM system, thus generating 31 procesess.

In the third step some maintenance processes were added to manage some aspects that are relevant for the Company business but that never were implemented by the Company, such as Planning ordinary maintenance's intervention, Human resources management, Warehouse management with spare parts and consumables, Personnel qualification management to meet normative requirements, Corrective maintenance management (ticket opening, see Figure 4), Predictive maintenance management (specifically inspections, see Figure 5), Asset status management, Service level management, Maintenance on the field and Logistics management.

\section{Key Performance Indicators}

The section in which KPIs have the biggest role is the wind turbine maintenance and the most influencing factors are certainly economical, technical and organizational. Maintenance performance indicators reflect achievement and progresses in meeting a goal; clearly, the greater is the installed capacity the higher are Operational and Maintenance costs.

Leading indicators measure performance before the maintenance process results starts to follow a particular trend and monitor if maintenance activities are producing good results in a long-term period. An example Preventive Maintenance (PM) Completion Rate: a low completion rate for PM would generate an increasing in asset maintenance work while a high completion rate means that asset preventive maintenance request is correctly being completed and, probably, future corrective maintenance requests will be reduced. Another example is the Outage Schedule Compliance an important metric to track future maintenance work because it allows to measure deferred asset maintenance, resulting in an increased risks and likelihood that asset performance will decrease at a future time, leading to lower capacity, increased downtime, and greater expenses.

Lagging indicators use historic data to obtain a measure to confirm coherence with long-term performance trends; they are used to determine how well a process performs.

In order to increase maintenance performance, both internal and external factors of a company should be considered as complex activities. Therefore, considering each influencing factor is essential to assess, control, measure and compare performances. The KPIs in technical standards (specifically UNI EN 15341: 2007) can be grouped into three categories: economical (E, 21 KPIs), technical (T, 21 KPIs) and organizational (O, $26 \mathrm{KPIs})$. A selection of them was considered to be linked to the maintenance related processes implemented as shown in Table III. These allow the Company to calculate performances of related processes.

\section{CONCLUSIONS AND FUTURE WORK}

Introducing BPMN 2.0 into an asset management model is an efficient way for controlling and sharing information between all actors involved in a process. Specifically, in maintenance management of wind turbines there are a lot of factors (e.g. weather conditions, personnel availability) and events such as logistics, administrative or technical ones (e.g. failure) that can change in an unpredictable way the process performances. The developed WEAMS BPM system allows the definition and execution of management processes within renewable energy power plant, simplifying relations among company functions, introducing standard activities for each process, assigning tasks to users, eliminating non added value phases thus providing an overall reduction of downtime of wind turbines, procurement optimisation due to higher efficiency in warehousing, human resources management, maintenance cost reduction.

\section{ACKNOWLEDGMENT}

This work was partially supported by WEAMS $\mathrm{N}$. F/050145/00/X32 project.

\section{REFERENCES}

[1] The Institute of Asset Management, "IAM asset management maturity guide v1.1," The Institute of Asset Management, Tech. Rep., Jun 2016. [Online]. Available: http://www.theiam.org/

[2] N. Trapani, M. Macchi, and L. Fumagalli, "Risk driven engineering of prognostics and health management systems in manufacturing," IFACPapersOnLine, vol. 48, no. 3, pp. 995 - 1000, 2015, doi: 10.1016/j. ifacol.2015.06.213. 


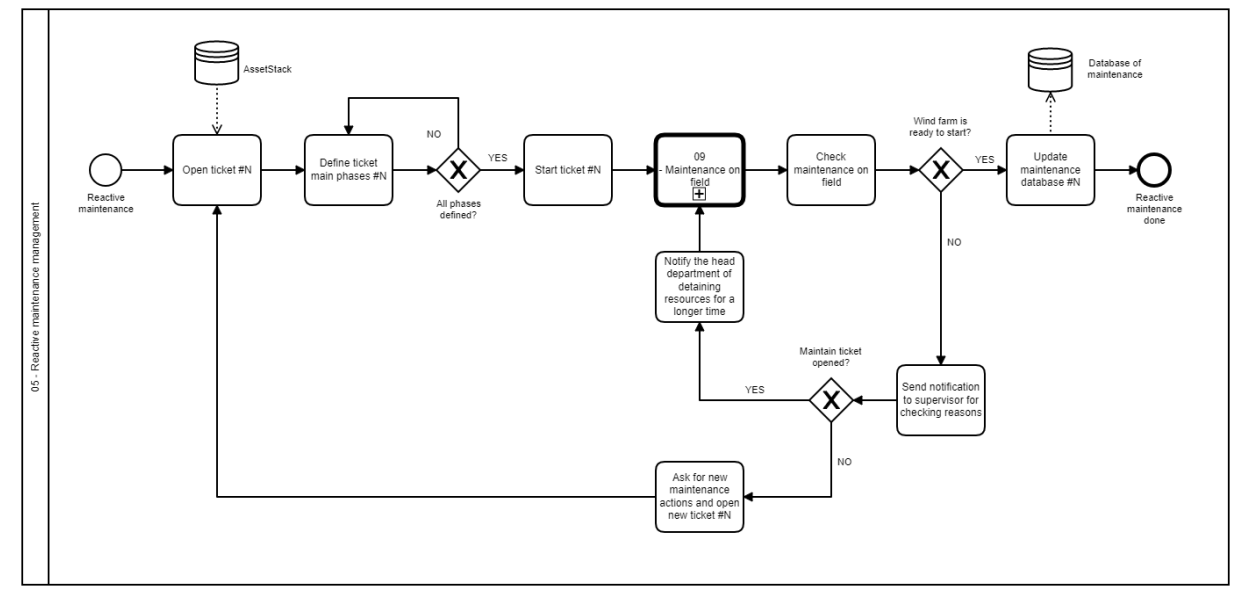

Fig. 4. Corrective Maintenance Management Process

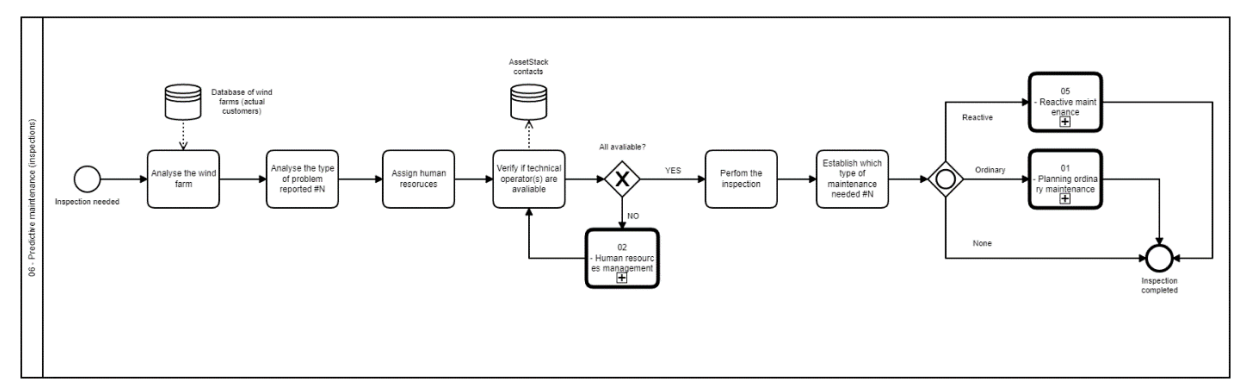

Fig. 5. Predictive Maintenance Management Process (inspection)

TABLE III

SElected KPIs \& Related Processes

\begin{tabular}{l|l|l} 
Type & Factors & Related processes \\
\hline E1 & Total maintenance costs / Asset, replacement value & $01 ; 02 ; 03 ; 04 ; 05 ; 06 ; 07 ; 09 ; 10$ \\
E3 & Total maintenance cost / Quantity of output & $01 ; 02 ; 03 ; 05 ; 06 ; 07 ; 08 ; 09 ; 10$ \\
T1 & Total Operating time / Total Operating time + Maintenance Downtime & $01 ; 05 ; 06 ; 07 ; 08 ; 09$ \\
T8 & Preventive maintenance time causing downtime / Maintenance Total downtime & $06 ; 09$ \\
O1 & Number of internal maintenance personnel / Total internal employees & $01 ; 02 ; 05 ; 06 ; 09$ \\
O2 & Number of indirect maintenance personnel / Number of internal maintenance personnel & $01 ; 02 ; 05 ; 06 ; 09$ \\
O3 & Number of indirect maintenance personnel / Number of direct maintenance personnel & $01 ; 02 ; 05 ; 06 ; 09$ \\
O16 & Corrective maintenance man-hours / Total maintenance man-hours & $01 ; 05 ; 06 ; 09$ \\
O18 & Preventive maintenance man-hours / Total maintenance man-hours & $01 ; 05 ; 06 ; 09$ \\
O22 & Work orders performed as scheduled / Total scheduled work orders & $01 ; 02 ; 05 ; 09$
\end{tabular}

[3] J. Montilva, J. Barrios, I. Besembel, and W. Montilva, "A business process model for it management based on enterprise architecture." CLEIej online vol.17, n.2, pp. 4-4, 08 2014, doi: 10.19153/cleiej.17.2.3.

[4] W. M. van der Aalst, M. La Rosa, and F. M. Santoro, "Business process management. don't forget to improve the process!" Business \& Information Systems Engineering 58(1), pp. 1-6, 01 2016, doi: 10.1007/s12599-015-0409-x.

[5] T. Allweyer, BPMN 2.0: Introduction to the Standard for Business Process Modeling. Books on Demand, 2016. [Online]. Available: https://books.google.it/books?id=sowaDAAAQBAJ

[6] M. Jasiulewicz-Kaczmarek, R. Waszkowski, M. Piechowski, and R. Wyczolkowski, "Implementing BPMN in maintenance process modeling," Advances in Intelligent Systems and Computing, vol. 656, pp. 303-309, 01 2018, doi: 10.1007/978-3-319-67229-8_27.

[7] Gartner, "Magic quadrant for intelligent business process management suites," Gartner, Tech. Rep., 2019, accessed 10 May 2019. [Online]. Available: https://www.gartner.com/en/documents/3899484

[8] I. Corporation, "Understanding the impact and value of enterprise asset management." https://www.ibm.com/downloads/cas/XJRD7M1Z, (c)IBM Corporation, Tech. Rep., 2016, accessed 10 May 2019.

[9] Accenture, "The future of onshore wind operations and maintenance," Accenture, Tech. Rep., 2017, accessed 10 May 2019. [Online]. Available: https://www.accenture.com/us-en/ insight-future-onshore-wind-operations-maintenance

[10] M. Shafiee and J. D. Sørensen, "Maintenance optimization and inspection planning of wind energy assets: Models, methods and strategies." Reliability Engineering and System Safety, pp. 1-19, 2017, doi: 10.1016/j.ress.2017.10.025.

[11] J. Wang, X. Zhao, and X. Guo, "Optimizing wind turbine's maintenance policies under performance-based contract." Renewable Energy, Volume 135, pp. 626-634, 05 2019, doi: 10.1016/j.renene.2018.12.006.

[12] A. Ciaramella, M. G. Cimino, B. Lazzerini, and F. Marcelloni, "Using bpmn and tracing for rapid business process prototyping environments." in ICEIS 2009 - 11th International Conference on Enterprise Information Systems, Proceedings, 01 2009, pp. 206-212, doi: 10.5220/ 0002005002060212. 